\title{
SUSTAINABLE CLUSTERING OF CUSTOMERS USING CAPACITIVE ARTIFICIAL NEURAL NETWORKS: A CASE STUDY IN PEGAH DISTRIBUTION COMPANY
}

\author{
Saeed Yousefi ${ }^{1}$, Hadi Shabanpour ${ }^{2}$ and Reza Farzipoor Saen ${ }^{3, *}$
}

\begin{abstract}
To survive, organizations should inevitably work based on sustainability principles in an ever-increasing changes of markets. Appropriate flexibility and responsiveness are particularly important when considering sustainability issue and market changes in clustering problem. One of the uses of clustering can be allocation of resources and equipment for providing the highest level of customer service which has been a matter of concern for decision makers in distributive companies. Capacitive clustering is a common method for solving allocation and distribution problems. However, traditional clustering models ignore sustainability criteria in defining clusters' capacity. The objective of this study, therefore, is to propose a novel method for optimizing resource allocation for customers given the sustainability criteria. Capacitive clustering is a technique that has a widespread application in data mining. This approach has been used for equipment distribution, sales targeting, market segmentation, etc. One prevalent clustering method is growing neural gas network (GNGN) technique. GNGN is a neural network with uncontrolled learning. In this paper, for the first time, we utilize GNGN to cluster customers given sustainability criteria. Here, the clusters' centers are determined and allocated with regard to capacity constraints of the clusters. The obtained results in general can be regarded as an optimized sustainable distribution system in which the number of trucks, distribution routes as well as fuel consumptions and environmental pollutions are minimized. We can also refer to reductions in urban traffic, maintenance costs, staff costs, and decreases in the fatigue of drivers and distributers due to the proximity of supermarkets. An illustrative case study is done to indicate the applicability and remarkable contributions of the suggested clustering approach.
\end{abstract}

Mathematics Subject Classification. 68T07.

Received October 16, 2019. Accepted May 31, 2020.

\section{INTRODUCTION}

Today, more than ever, an urgent necessity has arisen as human and organizational activities should be in line with sustainability principles. Every day, we hear alarming news from media regarding urban air pollution,

Keywords. Sustainable clustering, customers' clustering, distribution companies, capacitive artificial neural network, neural gas network (NGN).

1 SAP, Software Company, Tehran, Iran.

2 Young Researchers and Elite Club, Karaj Branch, Islamic Azad University, Karaj, Iran.

3 Faculty of Business, Sohar University, Sohar, Oman.

*Corresponding author: farzipour@yahoo.com 
crucial conditions of natural resources, environmental damage and occupational burnout caused by everincreasing operations of businesses [15]. Hence, public pressures on businesses are pushing them to obey and respect sustainability principles more and more. That is why not only environmental accountability but also social responsibility has become a significant duty of industries and businesses [18]. In fact, sustainability principles have been emerged as a key enabler for organizations to focus on alleviating environmental damages and enhancing social responsibility level [16]. The decision maker accordingly should consider social responsibility, environmental, and economic criteria while carrying out business activities, simultaneously [10]. Besides, the allocation and evaluation process has become more and more complicated with social and environmental criteria [29]. As a result, applying a reliable clustering model is necessary to effectively deal with the complexity of both sustainability and economic criteria.

On the other hand, market changes are extensively affecting the interactions of customers with organizations. Basically, ever-increasing "changes and interactions" are two main features of organizations' internal and external environments in new business era. The more changes in organizations, the more fluctuations in business environment [23]. This is due to the fact that more and more actors are playing role in the business environment which makes the environment more than ever competitive. Hence, to survive, organizations should be quite flexible to face with these alterations. Such flexibility can be realized by wise distribution and allocation of resources and equipment to customers, based on the recognition of the nature and volume of the changes [13]. Accordingly, an interesting aspect of this research is to design and propose a sustainable allocation/distribution system as a competitive advantage for distributing companies. In such a sustainable distribution system, not only economic costs are minimized but also, from the sustainability point of view, organizations would be more competitive in the market. As a result, we can expect reductions in the number of trucks, distribution routes as well as fuel consumptions and environmental pollutions. We can also refer to decreases in urban traffic, maintenance costs, staff costs, and decreases in the fatigue of drivers and distributers due to the proximity of supermarkets.

Some examples of allocation of resources and equipment in distributive companies include planning distribution paths in distribution companies, clustering customers, allocating research and development budget, and setting sales targets. As mentioned, the daily changes in volume, weight, and number of orders in different urban areas indicate a dynamic environment in defining the allocation problem. In such a dynamic atmosphere, usage of "clustering approach" is much more efficient and applicable compared to the other methods [25]. To do so, many clustering studies have been conducted. Among which, artificial neural network (ANN) has a special position and widespread applicability [25]. Furthermore, "data mining" is a key approach for clustering customers. One widespread and applicable "data mining" method has been "capacitive (capacity-based) clustering". This technique is used in cluster analysis approaches that can be implemented in various areas such as vehicle navigation, political zoning, market segmentation, and so on [13]. Nevertheless, common clustering techniques ignore sustainability criteria in defining clusters' capacity. Meaning that, for example, there are some sustainability aspects in defining constraints such as volume, weight, and number of orders that have not been applied in traditional clustering models. Hence, the common clustering techniques need to be developed in terms of the capacity issue. To overcome this shortcoming, here, a "capacity-based or capacitive clustering" model is developed.

The objective of this study is to optimize allocation of resources and facilities for customers (supermarkets). This study utilizes ANN approach to cluster customers using sustainability factors. Here, environmentally friendly and socially responsible ways of allocations are considered. To do so, a load allocation model (Capacitive Clustering Method) is proposed for a number of urban transport vehicles (trucks) which distribute products of Pegah Distribution Company in Tehran. Clustering demand points, in the modelling phase is determined based on "close distances" (the proximity of distribution points). By so doing, an acceptable pattern of clusters of customers can be designed in a way that each cluster is assigned to a truck. In summary contributions of our proposed approach are as follows:

- For the first time, simultaneous use of sustainability criteria (environmental, social, and economic criteria) in customers' clustering.

- The use of clustering in optimizing operations of capillary distribution of high demand goods. 
- Development of clustering model with simultaneous use of positive and negative criteria.

- For the first time, sustainable capacity creation for clustering in growing neural gas network (GNGN) algorithm.

- A case study is given to show applicability of the proposed model.

The rest of this paper is organized as follows: Literature review is presented in Section 2. In Section 3, proposed method is given. A case study is presented in Section 4. Finally, concluding remarks are discussed in Section 5 .

\section{Literature REVIEW}

\subsection{Sustainability, organizations, and customers}

The concept of sustainability, from an organizational point of view, refers to considering three environmental, social responsibility, and economic criteria, simultaneously [1]. To enhance customers' awareness regarding sustainability, Ting et al. [27] studied green customers of a Taiwanese hotel. Hadi et al. [9] investigated the relationship between sustainable service quality and customer loyalty in the Pakistan cellphone industry. By designing a sustainable reverse logistics network, Cinar [3] developed a mixed integer linear programming (MILP) framework to determine a long-term strategy. Likewise, Shabanpour et al. [24] evaluated and ranked sustainable suppliers using congestion approach of economies of scale theory of DEA. Another research work was carried out by Tavana et al. [26] in an attempt to assess sustainable suppliers, merging goal programming and dynamic DEA. They evaluated efficiency of sustainable suppliers over several periods. Furthermore, Yousefi et al. [28] developed a scenario-based robust DEA model to assess sustainable suppliers.

\subsection{Artificial neural networks (ANN)}

ANN is a non-linear function inspired from human's real neural network system [23]. Nowadays, ANN has many applications such as clustering [7,19,25,29], classification [12], and ranking [12,14]. The ANN has also been applied to forecast future dataset $[11,17,23]$. Using cluster analysis and DEA-discriminant analysis, Farzipoor Saen [5] predicted group membership of new customers. Recently, Yousefi et al. [29] applied ANN clustering techniques to classify Japanese fossil fuel power plants in appropriate clusters. Then, they ranked the plants in each cluster. They classified the plants in different clusters according to their similarity. As a result, the rankings in each cluster are based on pairwise comparisons of similar power plants [29].

\subsection{ANN's applications in capacitive clustering}

Numerous clustering techniques have been used for dealing with big data analysis [2]. However, considering capacity for clusters was a mean of enhancing reliability of results of clustering [7]. Capacitive (capacity-based) clustering includes a number of clusters with capacity constraints and maximum possible similarity in the cluster [21]. When the basis for comparing clusters is Euclidean distance, the p-median clustering model responds better in some data structures. Hence, in many cases, "capacitated clustering" issues are modeled in the form of a p-median problem [7]. As addressed by Shamsipoor et al. [25], various methods have been developed for solving p-median capacitated problems such as using branch and bound and Lagrange Liberation (LL) and applying neural networks [4] and genetic algorithms [8].

To solve capacitated clustering problem, Shamsipoor et al. [25] applied self-organized mapping (SOM) neural networks. SOM is an uncontrolled learning neural networks technique which has been widely used in clustering problems [7]. Shamsipoor et al. [25] used a special type of neural networks called "neural gas networks". Subsequently, Ghesmoune et al. [7] discovered the hidden topology in the data using the advantage of study of Shamsipoor et al. [25]. They designed an initial clustering model and then entered the operational constraints of the distribution of goods in the initial results [7]. Using a linear allocation model, then, they completed the results of the GNGN and converted initial clustering model into a "capacitated clustering" one. 
TABLE 1. Notations.

\begin{tabular}{ll}
\hline \hline Notations & Descriptions \\
\hline$w_{a}$ & Random neuron $(a=1, \ldots, A)$ \\
$w_{b}$ & Random neuron $(b=1, \ldots, A)$ \\
$\xi$ & Input signal \\
$P$ & Number of input signals $(p=1, \ldots, P)$ \\
$s_{1}$ & The nearest neuron $(s=1, \ldots, S)$ \\
$s_{2}$ & The nearest next neuron $(s=1, \ldots, S)$ \\
$\epsilon_{b}$ & Transition ratio \\
$\epsilon_{n}$ & Transition ratio \\
$\alpha_{\max }$ & All edges with the greatest age of $\alpha(a=1, \ldots, A)$ \\
$\lambda$ & Integer multiplication \\
$r$ & Error \\
$f$ & Neuron with the largest error $(f=1, \ldots, F)$ \\
$q$ & New neuron with maximum accumulated error $(q=1, \ldots, Q)$ \\
$\alpha$ & A fixed multiplier for reducing error between $q$ and $f$ \\
$d$ & A fixed multiplier for reducing all error values \\
\hline
\end{tabular}

\subsection{Knowledge gap}

Although SOM has significant potential for solving clustering problem, they use a default topology [20]. Indeed, the SOM neural networks need presumptive topology which should be characterized by cluster topology before they can be implemented [7]. This can be difficult if cluster topology is unclear. Hence, another model of neural networks called neural gas network (NGN) was developed to explore topology during problem solving. NGN is a simple neural network. NGN performs optimal data representation based on feature vectors. This algorithm does not use any presumptive topology [22]. One of the versions of NGN is GNGN. In GNGN, a small primary network is considered, and new neurons are added by estimating local statistical quantities from the previous matching steps [6]. However, common clustering techniques ignore sustainability criteria in defining clusters' capacity. For example, there are some sustainability aspects in constraints such as volume, weight, and number of orders that cannot be applied in traditional clustering models. To overcome this shortcoming, for the first time, we develop a sustainable GNGN framework for clustering customers. Here, a capacitive clustering GNGN model is developed to deal with the complexity of sustainability criteria.

\section{RESEARCH METHOD}

\subsection{Proposed steps}

The main steps of our algorithm include data collection, initial customers' clustering, and mathematical modeling. In the initial clustering, centers of primary clusters are identified. In the next step, using an integer linear model, the maximal capacity of each cluster is determined, and each cluster is adjusted according to their default capacity. To analyze the primary cluster and find cluster centers, we use an algorithm which is called GNGN grid. At this juncture, as is depicted in Table 1, notations of the GNGN algorithm are defined.

Based on the model proposed by Fritzke [6], the GNGN algorithm includes the following steps:

(1) Starting the algorithm by creation of two random neurons $w_{a}$ and $w_{b}$.

(2) Generation of an input signal $\xi$ based on $P(\xi)$.

(3) Finding the nearest neuron $s_{1}$ and the nearest next neuron $s_{2}$.

(4) Increasing the age of all the edges resulted from $s_{1}$. 
(5) Calculation of the square distance between the input signal and the nearest neuron in the input space.

$$
\Delta\left(s_{1}\right)=\left\|w_{s_{1}}-\xi\right\| .
$$

(6) Transition of $s_{1}$ and its direct neighbors to $\xi$ by the ratios $\epsilon_{b}$ and $\epsilon_{n}$.

$$
\begin{aligned}
\Delta w_{s_{1}} & =\epsilon_{b}\left(\xi+w_{s_{1}}\right) \\
\Delta w_{n} & =\epsilon_{n}\left(\xi+w_{n}\right) .
\end{aligned}
$$

(7) If $s_{1}$ and $s_{2}$ are connected by an edge, the age of the edge is set equal to zero, and if there is no such edge, it should be created.

(8) All edges with age of greater than $\alpha_{\max }$ are deleted. If they do not lead to create any edges, they are also deleted.

(9) If all the input signals generated so far are integer multiple of the parameter $\lambda$, a new neuron is created which has the following features:

- The new neuron which is called $q$ has maximum accumulated error.

- The neuron $r$ is put between neuron $q$ and its neighbor with the largest error $(f)$

$$
w_{r}=\frac{1}{2}\left(w_{q}+w_{f}\right) .
$$

- The edge is put between neurons $r$ and neurons $q$ and $f$, in addition to removing the main edge between $q$ and $f$.

- Reduction of error between $q$ and $f$ with multiplication of them in the fixed value of $\alpha$.

- Estimation of initial amount of error $r$ with new value of error $q$.

(10) Decrease all error values by multiplication in the fixed value of $d$.

(11) If the stop indicator (network size or some performance indicators) is not applicable, then again, proceed from step one.

\subsection{Mathematical modeling}

Assuming that the cluster centers are calculated with the growing algorithm of the NGN, the following proposed model can be used to determine the membership of each sample in each cluster. This model is a linear allocation model and the logic of the objective function is to minimize the distances between the samples and the corresponding cluster centers. In this model, two sets are considered. The Pnt set which contains demand points and the Cls set that consists of cluster centers. Both sets are derived from the running of the growing NGN. In other words, assuming $n$ samples and $k$ clusters, we have:

$$
\begin{array}{ll}
\operatorname{Pnt}=\left\{p_{1}, p_{2}, \ldots, p_{n}\right\}, & \mathrm{Cls}=\left\{c_{1}, c_{2}, \ldots, c_{n}\right\} \\
\min \sum_{i} \sum_{j} d_{i j} x_{i j} & \\
\text { s.t. } & \forall i \in \mathrm{Pnt} \\
\sum_{j} x_{i j}=1 & \forall i \in \mathrm{Cls} \\
\sum_{i} x_{i j} \leq n & \forall i \in \mathrm{Cls} \\
\sum_{i} w_{i} x_{i j} \leq w & \forall i \in \mathrm{Cls} \\
\sum_{i} v_{i} x_{i j} \leq v & \forall i \in \mathrm{Pnt}, \quad \forall i \in \mathrm{Cls} . \\
x_{i j} \epsilon\{0,1\} &
\end{array}
$$


The equation (3.1) shows the minimization of the target function, in which $x_{i j}$ indicates the allocation of the sample $i$ to the cluster $j$. According to mathematical expression (3.4), if the sample $i$ is assigned to the cluster $j$, the $x_{i j}$ is equal to 1 ; otherwise it is equal to 0 . Here, $d_{i j}$ specifies the distance between sample $i$ and the center of the cluster $j$. This variable determines the cost of assigning the desired sample to a given cluster. Expressions (3.2)-(3.4) represent optimization constraints. Expression (3.2) guarantees that each sample is assigned to only one cluster. The constraints in (3.3)-(4.1) indicate the number cap, the weight cap, and the volume cap. Here, the same cap is considered for the constraints in all clusters which are displayed by $n, v$, and $w$, respectively. $w_{i}$ and $v_{i}$ also show the weight and sample size of $i$, respectively. As is seen, the above model is an integer linear programming model. By solving this, the allocation of each sample (distribution points) is obtained in each cluster.

\section{Case study And Data Analysis}

\subsection{Introduction of the SAP software company}

SAP Company, as a knowledge-based organization has been working in the field of developing the Enterprise Resource Planning (ERP) software. SAP's distinguished ERP software is result of consideration and implementation of the views of elites and experts in the manufacturing and distribution industries. There are two competitive advantages for SAP Company. SAP enjoys several years of experience of its elites, and; the ability of customizing the world's software science. These advantages have created a clear distinction with other similar software products.

SAP wishes to design and determine the optimal distribution routes for Pegah Distribution Company in Tehran (Iran) province (as one of the SAP customers). There are 354 supermarkets. The demands of the points have a specific weight and volume. The demands of the points are collected over a period of time along with their geographical coordinates. The problem is to determine the minimum number of vehicles (trucks) that can cover all of these points in one day, by considering the constraints of number, weight, and volume, simultaneously.

\subsection{Defining economic, environmental, and social criteria}

The problem turns into a sustainable capacitated clustering problem of customers (supermarkets). Each cluster can have at most 40 orders, $2500 \mathrm{~kg}$, and 4 cubic meters, respectively. Each cluster represents a "distribution truck" which can serve the supermarkets by taking into account the cap of number of orders, weight, and volume. The "total weight of the demand of all supermarkets" and the "total volume of the demand of all supermarkets" per day are $27560 \mathrm{~kg}$ and 56 cubic meters, respectively. The total number of supermarkets is 354. By dividing each of these three numbers (constraints) on their cluster cap, we get at least 11, 12, and 13 required clusters. This minimum clustering is based on the number (8.85), weight (11.02) and volume (13.87). Therefore, the minimum number of clusters is 14 .

From the environmental aspect, if the order weight is over $2500 \mathrm{~kg}$, the fuel consumption and pollutions are increased. Therefore, this factor is considered as an environmental criterion, and the excess weight in truck loading should be avoided in modeling. Besides, to use the maximum truck capacity, 4 cubic meters of truck volume is considered as an economic factor. Also, according to the experts' opinion, 40 orders are given per truck in line with the social responsibility of the organization. This order quantity is based on the work hours allowed for each distribution worker, the delivery time of each order, the distance between each order, and the arriving time of the first order.

Note that using the proposed "order clustering approach" leads to loading the nearest-distance orders on one truck. In addition, our clustering approach considers economic, social, and environmental criteria, simultaneously; as firstly, with the optimum number of trucks, the maximum order distribution is carried out (economically). Secondly, trucks travel the shortest distance which means consuming the least fuel (environmental). Thirdly, no more than the allowed size of orders (40 orders) is assigned to each distribution team. From the aspect of labor health and safety (social responsibility), this avoids distribution workers from feeling exhausted and pressured. 
TABLE 2. Centers of clusters derived from the GNGN.

\begin{tabular}{lll}
\hline \hline Clusters & Latitude & Longitude \\
\hline 1 & 35.7296251398067 & 51.1322282513600 \\
2 & 35.7790509622515 & 51.4624222749587 \\
3 & 35.7513331077846 & 51.2886564100309 \\
4 & 35.6320912965813 & 51.4292430356884 \\
5 & 35.5596138548713 & 51.2452579811807 \\
6 & 35.7709638881209 & 51.3787713999846 \\
7 & 35.6778320123794 & 51.3832760676116 \\
8 & 35.6384623521130 & 51.3535257541872 \\
9 & 35.7185241607981 & 51.4221860588045 \\
10 & 35.7184291845464 & 51.3284989093821 \\
11 & 35.5580417175905 & 51.4579669656753 \\
12 & 35.6760063971082 & 51.4838051516794 \\
13 & 35.7304362621130 & 51.5249400300322 \\
14 & 35.7532035816614 & 51.2452579811807 \\
\hline
\end{tabular}

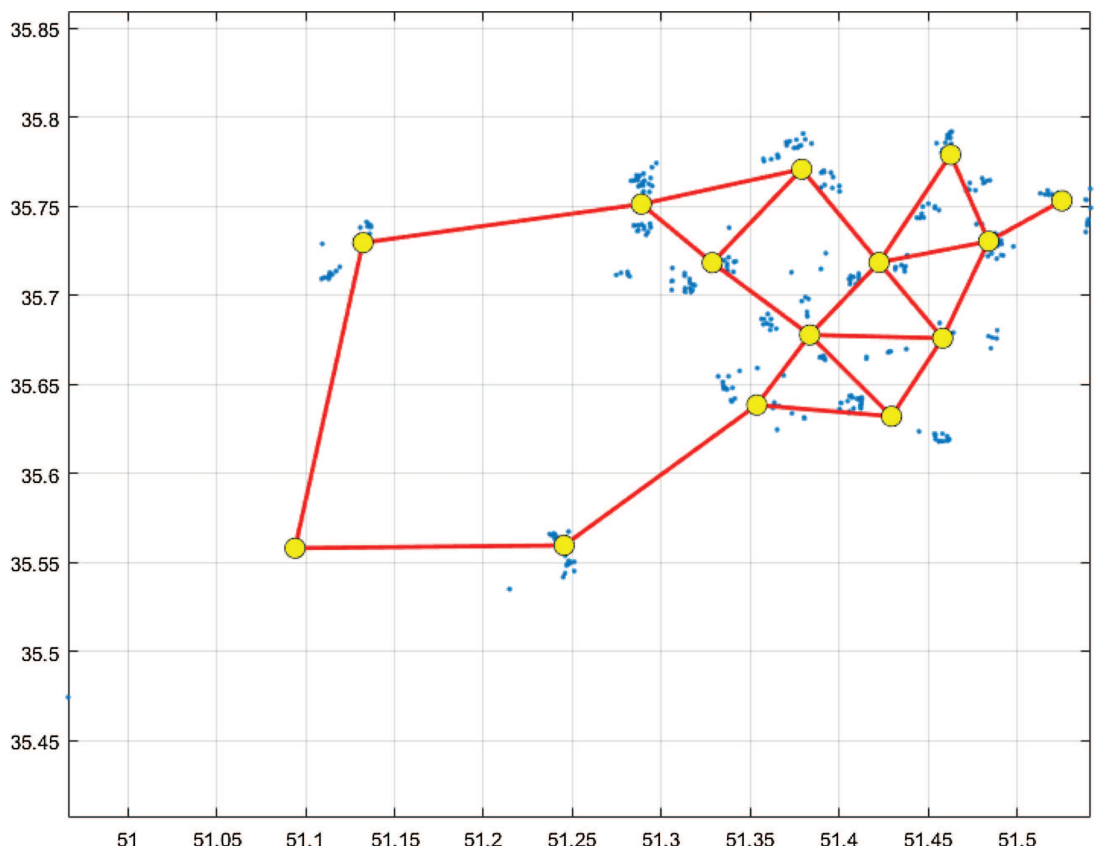

FigURE 1. Results derived from the GNGN algorithm.

In the first step, 354 supermarkets are split into 14 clusters using GNGN algorithm in 200 replications. Input vectors participate in GNGN training with two indicators of latitude and longitude coordinates. In implementing the algorithm, the parameters $\epsilon_{b}, \epsilon_{n}$, and $\alpha_{\max }$ are considered as $0.2,0.005$, and 50, respectively. The obtained clusters' centers are shown in Table 2. In Table 2, each row contains the latitude and longitude of a center. Each center represents the area in which the "truck" should distribute its products.

In addition to Table 2, the centers of the 14 clusters along with the supermarkets are shown in Figure 1. Relationships among centers are based on neighborly relations derived from the GNGN algorithm. 


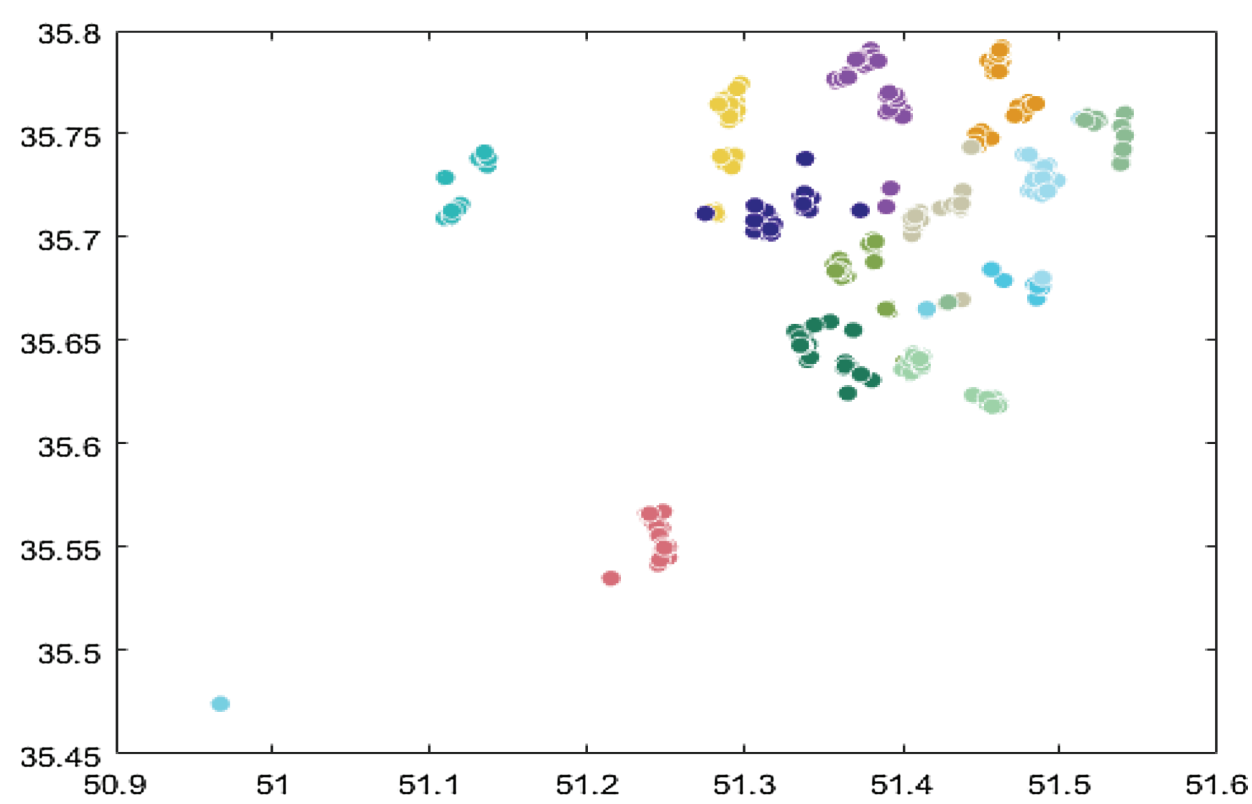

FiguRE 2. Ultimate capacitated clustering.

After running GNGN, the integer linear allocation model (introduced in the previous section) is run. The model given the parameters is specified below:

$$
\begin{array}{ll}
\min \sum_{i} \sum_{j} d_{i j} x_{i j} & \\
\text { s.t. } & \forall i \in \text { Pnt } \\
\sum_{j} x_{i j}=1 & \forall i \in \mathrm{Cls} \\
\sum_{i} x_{i j} \leq 40 & \forall i \in \mathrm{Cls} \\
\sum_{i} w_{i} x_{i j} \leq 2500 & \forall i \in \mathrm{Cls} \\
\sum_{i} v_{i} x_{i j} \leq 4 & \forall i \in \mathrm{Pnt}, \quad \forall i \in \mathrm{Cls} . \\
x_{i j} \in\{0,1\} &
\end{array}
$$

All parameters are specified and the matrix $D=\left[d_{i j}\right]$ is also obtained based on the calculation of the intervals between each of the points and each of the cluster's centers derived from GNGN. To solve the above problem, we run MATLAB software using the "intlinprog" function. The results indicate that which supermarket should be assigned to which cluster.

In Figure 2, the final results of the capacitated clustering are presented. As is seen, 354 supermarkets are divided into 14 clusters which are marked in 14 different colors. Based on the sustainability constraints of the model, our clustering approach ensures that none of the trucks exceeds their loading capacity. 


\section{Conclusions And ReCOmmendations}

Nowadays, organizations wish to gain competitive advantages to effectively adopt the business changes. Capacity-based clustering is a widespread approach in data mining to deal with the changes [13]. This study proposed a novel sustainable capacitated clustering approach for optimally allocation and distribution of goods. To do so, we utilized sustainability criteria and ran the growing NGN and linear allocation model. By so doing (as is seen in Fig. 2), 354 supermarkets are divided into 14 clusters. Based on the sustainability constraints, our clustering approach ensures that none of the delivery trucks exceeds their loading capacity and the nearest-distance orders are loaded on one truck. Besides, the clustering approach considers economic, social, and environmental criteria, simultaneously. Meaning that, firstly, the maximum distribution orders are delivered to customers via the optimum (minimum) number of trucks (economic aspect). Secondly, this would also be remarkable from environmental viewpoint as the trucks consume the least amount of fuel and emit less air pollution with minimized traffic on streets and shortest distances. In addition, given the specified capacity of each truck's load $(2500 \mathrm{~kg})$, we also expect minimum fuel consumptions and environmental pollutions. Thirdly, no more than the allowed size of orders (40 orders) is assigned to each distribution staff. From the labor health and safety aspect (social responsibility), this reduces workers fatigue.

\subsection{Managerial implications}

From managerial viewpoint, two main advantages would be expectable as optimized delivery times and transport costs. Utilizing this approach in distribution companies can lead to reducing urban traffic, shortening transport routes, decreasing maintenance costs, reducing staff numbers and costs, decreasing number of delivery trucks, and reducing the fatigue of drivers and distributers due to the proximity of supermarkets. This sustainable capacitated clustering method can also be utilized for solving relevant problems of other distribution companies.

\subsection{Future research directions}

Given the results of this research, it is possible for researchers to apply self-organized mapping (SOM) neural networks instead of the NGN technique to compare the final results with each other. Another interesting research topic could be adding topology limitations to the allocation problem.

Acknowledgements. Authors would like to appreciate three Reviewers for their constructive comments.

\section{REFERENCES}

[1] A. Amindoust, Supplier selection considering sustainability measures: an application of weight restriction fuzzy-DEA approach. RAIRO:OR 52 (2018) 981-1001.

[2] H. Amoozad Mahdiraji, E. Kazimieras Zavadskas, A. Kazeminia and A.A. Abbasi Kamardi, Marketing strategies evaluation based on big data analysis: a CLUSTERING-MCDM approach. Econ. Res. 32 (2019) 2882-2898.

[3] S. Cinar, Sustainable reverse logistic network design for End-of-Life Use-Case Study. To appear in: RAIRO:OR (2019). DOI: $10.1051 / \mathrm{ro} / 2019069$

[4] E. Domínguez and J. Muñoz, A neural model for the p-median problem. Comput. Oper. Res. 35 (2008) $404-416$.

[5] R. Farzipoor Saen, Using cluster analysis and DEA-discriminant analysis to predict group membership of new customers. Int. J. Bus. Exc. 6 (2013) 348-360.

[6] B. Fritzke, A growing neural gas network learns topologies. In: Proceedings of the 7th International Conference on Neural Information Processing Systems. Denver, CO (1995) 625-632.

[7] M. Ghesmoune, M. Lebbah and H. Azzag, A new growing neural gas for clustering data streams. Neural Networks 78 (2016) $36-50$.

[8] K. Ghoseiri and S.F. Ghannadpour, Solving capacitated p-median problem using genetic algorithm. In: IEEE International Conference on Industrial Engineering and Engineering Management (2007) 885-889.

[9] N.U. Hadi, N. Aslam and A. Gulzar, Sustainable service quality and customer loyalty: the role of customer satisfaction and switching costs in the Pakistan cellphone industry. Sustainability 11 (2019) 1-17.

[10] A. Halog and N.H. Nguyen, Evaluating supply chain networks by incorporating the triple dimensions of sustainability paradigm, chapter 8. In: Implementing Triple Bottom Line Sustainability into Global Supply Chains, edited by L. Bals, W. Tate. Greenleaf Publishing in Association with GSE Research. Taylor \& Francis, London (2017) 165-186. 
[11] A. Ibiwoye, E. Ajibola and A.B. Sogunro, Artificial neural network model for predicting insurance insolvency. Int. J. Manage. Bus. Res. 2 (2012) 59-68.

[12] R. Krakovsky and R. Forgac, Neural network approach to multidimensional data classification via clustering. In: IEEE 9th International Symposium on Intelligent Systems and Informatics (2011) 169-174.

[13] A. Kumar, V. Jain and S.A. Kumar, A comprehensive environment friendly approach for supplier selection. Omega 42 (2014) $109-123$.

[14] R.J. Kuo, Y.C. Wang and F.C. Tien, Integration of artificial neural network and MADA methods for green supplier selection. J. Clean. Prod. 18 (2010) 1161-1170.

[15] Y. Li, R.J.S. Beeton, T. Sigler and A. Halog, Enhancing the adaptive capacity for urban sustainability: a bottom-up approach to understanding the urban social system in China. J. Environ. Manage. 235 (2019) 51-61.

[16] T. Lu and A. Halog, Towards better life cycle assessment and circular economy: on recent studies on interrelationships among environmental sustainability, food systems and diet. Int. J. Sustainable Dev. World Ecol. 27 (2020) $515-523$.

[17] P. Lu and M.S. Rosenbaum, Artificial neural network and grey system for the prediction of slope stability. Nat. Hazards $\mathbf{3 0}$ (2003) 383-398.

[18] L.Q. Luu and A. Halog, Life cycle sustainability assessment: a holistic evaluation of social, economic, and environmental impacts, chapter 14. In: Sustainability in the Design, Synthesis and Analysis of Chemical Engineering Process, edited by G. Ruiz-Mercado, H. Cabezas. Elsevier, Butterworth-Heinemann, Oxford, MA (2016) 327-352.

[19] C. Melchiorre, M. Matteucci, A. Azzoni and A. Zanchi, Artificial neural networks and cluster analysis in landslide susceptibility zonation. Geomorphology 94 (2008) 379-400.

[20] D.H. Milone, G. Stegmayer, L. Kamenetzky, M. López and F. Carrari, Clustering biological data with SOMs: on topology preservation in non-linear dimensional reduction. Expert Syst. App. 40 (2013) 3841-3845.

[21] M.J.N. Negreiros and A.W.C. Palhano, The capacitated centered clustering problem. Comput. Oper. Res. 33 (2006) $1639-1663$.

[22] R.S. Novin, M.T. Masouleh and M. Yazdani, A new neural gas network approach for obtaining the singularity-free workspace of 3-DOF planar parallel manipulators. Proc. Inst. Mech. Eng. Part C: J. Mech. Eng. Sci. 232 (2016) 174-189.

[23] H. Shabanpour, S. Yousefi and R. Farzipoor Saen, Forecasting efficiency of green suppliers by dynamic data envelopment analysis and artificial neural networks. J. Clean. Prod. 142 (2017) 1098-1107.

[24] H. Shabanpour, A. Fathi, S. Yousefi and R. Farzipoor Saen, Ranking sustainable suppliers using congestion approach of economies of scale theory of data envelopment analysis. J. Clean. Prod. 240 (2019) 118190.

[25] H. Shamsipoor, M.A. Sandidzadeh and M. Yaghini, Solving capacitated p-median problem by a new structure of neural network. Int. J. Ind. Eng. 19 (2012) 305-319.

[26] M. Tavana, H. Shabanpour, S. Yousefi and R. Farzipoor Saen, A hybrid goal programming and dynamic data envelopment analysis framework for sustainable supplier evaluation. Neural Comput. App. 28 (2016) 3683-3696.

[27] C.T. Ting, C.M. Hsieh, H.P. Chang and H.S. Chen, Environmental consciousness and green customer behavior: the moderating roles of incentive mechanisms. Sustainability 11 (2019) 819.

[28] S. Yousefi, H. Shabanpour, R. Fisher and R. Farzipoor Saen, Evaluating and ranking sustainable suppliers by robust dynamic data envelopment analysis. Measurement 83 (2016) 72-85.

[29] S. Yousefi, R. Soltani, A. Bonyadi Naeini and R. Farzipoor Saen, A robust hybrid artificial neural network double frontier data envelopment analysis approach for assessing sustainability of power plants under uncertainty. Expert Syst. 36 (2019) e12435. 